\title{
The Application Study of HHT for the Dynamic EEG Data under + Gz Acceleration
}

\author{
Yifeng $\mathrm{Li}^{*}$, Lihui Zhang, Quan Wang, Xiaoyang Wei, Hong Wang, Sanyuan Wu, Baohui Li and Rong Lin \\ Institute of Aviation Medicine, Air Force Beijing, 100142, China \\ "Corresponding author
}

\begin{abstract}
The basic law and method of Hilbert-Huang Transformation (HHT) is introduced, and HHT is induced into the application study of dynamic EEG data under $+\mathbf{G z}$ acceleration. The dynamic EEG data at static state that is when $1 G$ and under $+\mathrm{Gz}$ acceleration is made time frequency analysis by using HHT method, and the analysis result is basically consistent with that of FFT, proving the feasibility of this method on dynamic EEG data analysis. It is found by contrast that, HHT is more appropriate for the analysis and treatment of nonstationary signal, moreover, the whole process is adaptive. HHT can better show the distribution of signal energy in time frequency domain by Hilbert spectrum and more clearly reflect the frequency spectrum change feature of dynamic EEG than FFT.
\end{abstract}

Keywords- HHT; dynamic EEG; frequency spectrum characteristic;

\section{INTRODUCTION}

In flying and under $+\mathrm{Gz}$ acceleration, the occurring of human syncope can inevitablely lead to the change of human physiological signals. By the analysis of human physiological signals and parameters under, extracting the valuable information signal contains can make monitoring and evaluation for human physical condition under $+\mathrm{Gz}$ acceleration. In the field of Aeronautics and Astronautics, in order to prevent pilots occurring air syncope in flying, many scholars have began to make monitoring and research for human physiological signals under $+\mathrm{Gz}$ acceleration[1][2]. Monitoring and analysis of physiological signals including EEG is one of the key technologies to determine if the state before human syncope can be judged, identified and early warning ahead of time. All kinds of analysis methods such as Fourier transformation[3][4][5],Short Time Fourier transformation[6], wavelet and wavelet package transformation[7][8][9][10][11][12][13][14][15] and other technologies have been applied to analysis of signal. Traditional Fourier transformation belongs to stable state analysis method, and can better treat frequency characteristic of stable state signal, but it not possesses time frequency resolving power at the same time and can not reflect exactly time varying characteristic of non stable state signal, and it requires analysis signal is stable state and linear, while EEG is non-stationary, nonlinear random signal. Short Time Fourier transformation is a kind of more natural time frequency analysis thinking, though Fast Fourier transformation possesses the advantages of arithmetic speed is faster and convenient use and others, the treatment effect for non- stationary, nonlinear signal is less than satisfactory. Furthermore, according to the Heisenberg uncertainty theorem, the product of time width and frequency band width of signal should be a constant which is associated with sampling rate. In other words, when it is needed to improve the accuracy of time domain, the accuracy of frequency domain often is needed to be sacrificed. In turn, that's the way. That is subjected to the restrict of uncertainty principle, it can not obtain a higher resolution in time domain and frequency domain at the same time. Wavelet transformation is three dimensional signal processing method have time frequency resolution ability simultaneously, and can provide localization information of time domain and frequency domain of signal at the same time, but its resolution is still subjected to the restrict of uncertainty principle, so the same signal adopting different wavelet basis functions may lead to that analysis results difference much, and it is difficult to make precise analysis of time and frequency domain, existing limitation in practical application. The HHT method is a new signal processing method proposed by Huang and others in 1998, which can analyze and treat signal in time frequency domain, and can make adaptive decomposition based on the local feature information of signal itself, not need to pre-set parameters. Therefore, it overcomes the problem of depending on the subjective experience of prediction personnel, and has stronger ability to resist noise, so can better reflect the essential characteristic of signal. This method has been favored by the majority of researchers as soon as it appears, and has been applied rapidly in the research of all kinds of fields such as geology, engineering, medicine and so on[16][17][18][19][20][21]. However, it has not been seen that this method is applied to the study of EEG and other biomedical signals. In this study, EEG data under centrifuge $+\mathrm{Gz}$ acceleration is first obtained, then HHT is induced to the application study of the dynamic EEG data under $+\mathrm{Gz}$ acceleration. The dynamic EEG data at static state that is when $1 \mathrm{G}$ and under $+\mathrm{Gz}$ acceleration is made time frequency analysis by using HHT method, and all kinds of spectrum graphs are made out, then the actual meaning of spectrum graphs and the change characteristic of dynamic $\mathrm{EEG}$ under $+\mathrm{Gz}$ acceleration is studied, proving the feasibility of HHT method in dynamic EEG data analysis.

\section{The BASIC PRINCIPLE AND METHOD[22]}

HHT is the process that it makes smoothly treatment for signal, and makes a stepwise decomposition of fluctuation of different scales in signal, formatting intrinsic mode function (IMF) components, then makes Hilbert transformation for 
these components. The concrete steps of empirical mode decomposition (EMD) are as followings:

(1)The local maximum and minimum values of the original data $x(t)$ are found out, and the maximum value envelope $x_{\max }(t)$ and the minimum value envelope $x_{\min }(t)$ are got separately by using the interpolation connection of 3 times spine curve.

(2)The instantaneous average value $m(t)$ is obtained by making an average of the local maximum value $x_{\max }(t)$ and the minimum value $x_{\min }(t)$ at each moment;

$$
m(t)=\left[x_{\max }(t)+x_{\min }(t)\right] / 2
$$

(3)A new sequence of losing low frequency $h(t)$ is obtained by that the original sequence $x(t)$ subtracts the instantaneous average value.

$$
h(t)=x(t)-m(t)
$$

For different data sequence, $h(t)$ maybe is intrinsic mode function (IMF), and also maybe is not it. Intrinsic mode function (IMF) must satisfy the following two conditions: (a)The number of extreme points and the number of crossing zero point is equal or differences 1 at most. (b) At any moment, the average value of two envelopes constituted by local maximum value points and local minimum value points is 0 .

The condition (a) is similar to the traditional narrow band requirement of the stationary Gauss process, and the condition (b) is to ensure that the instantaneous frequency obtained by IMF has meaning. Checking whether $h(t)$ satisfies the above

two conditions, if satisfying, then $h(t)$ is an IMF. If not satisfying, $h(t)$ is taken as the original sequence to repeat the above 3 steps, until satisfying the (a), (b) two conditions. So the first IMF is got, and is marked $C_{1}(t)$. In general, $C_{1}(t)$ is on behalf of the high frequency part of the original sequence, and $C_{1}(t)$ also is called as a mode of vibration of the original sequence. The difference value sequence $r_{1}(t)$ of removing high frequency components is got by the original sequence subtracting $C_{1}(t)$, and the second IMF component $C_{2}(t)$ can be got by that $r_{1}(t)$ is made the above the smooth treatment process, so repeated indefinitely until the last sequence $r_{n}(t)$ cannot be decomposed any more $\left(r_{n}(t)\right.$ is monotonic function or constant), thus this time $r_{n}(t)$ is on behalf of the mean value of the original sequence or trend. At last, the original data sequence is represented by these IMF components and a trend or average value:

$$
x(t)=\sum_{i=1}^{n} C_{i}(t)+r_{n}(t)
$$

The IMF obtained by EMD has the characteristics of narrow band, and is suitable for Hilbert transformation, so the instantaneous frequency and the Hilbert frequency spectrum can be obtained. In brief, Hilbert transformation is just the convolution of signal and $1 / \mathrm{t}$, so it emphasizes the local property, thus avoiding the high and low frequency parts which is produced by the forced fitting in Fourier transformation and does not exist in fact.

IMF is made Hilbert transformation:

$$
£(t)=\frac{1}{\pi} P V \int_{-\infty}^{\infty} \frac{C(\tau)}{T-\tau} d \tau
$$

In the formula, $\mathrm{P} \mathrm{V}$ is on behalf of Cauchy Principal Value, so the analytic signal of $C(t)$ is defined as:

$$
z(t)=C(t)+i £(t)=a(t) e^{i \theta(t)}
$$

In the formula

$$
\begin{aligned}
& a(t)=\left[C(t)^{2}+£(t)^{2}\right]^{1 / 2} \\
& \theta(t)=\tan ^{-1} \frac{£(t)}{C(t)}
\end{aligned}
$$

Instantaneous amplitude and instantaneous phase are expressed explicitly in the above formulas, well reflecting the instantaneous characteristic of sequence. On the basis, the defined instantaneous frequency is as:

$$
w(t)=\frac{d \theta(t)}{d t}
$$

It can be seen from the above that, the amplitude and frequency got by Hilbert transformation which is made for IMF are both functions of time. If the amplitude is displayed on frequency-time plane, the Hilbert spectrum of IMF $H(w, t)$ can be obtained. If it is made an integral of time, so the marginal spectrum can be obtained:

$$
h(w)=\int_{0}^{T} H(w, t) d t
$$

If its square is made an integral for $w$, so the instantaneous energy can be obtained: 


$$
I E(t)=\int_{w} H^{2}(w, t) d w
$$

If it is made an integral of time, so the energy spectrum can be obtained:

$$
E S(w)=\int_{0}^{T} H^{2}(w, t) d t
$$

HHT analysis no longer thinks that time and frequency is absolute variables of two rooms, and no longer thinks that the basic ingredient is complete sine function, that is in the basic ingredients, instantaneous frequency also changes with time. These are all something with enlightenment in the development of data treatment and signal analysis.

\section{THE APPLICATION AND ANALYSIS OF HHT IN DYNAMIC EEG DATA UNDER +GZ ACCELERATION}

\section{A. Experiment Data Acquisition}

The experimental data is acquired from 5 healthy volunteers. The subjects are all healthy, no human centrifuge contraindications. The main equipment is domestic, new type human centrifuge, and subjects are exposed under $+\mathrm{Gz}$ acceleration according to the set curve. The set curve starts from $1 \mathrm{G}$, reaches the maximum $\mathrm{G}$ value at a certain growth rate, lasting for 10-15 seconds, and then drops to $1 \mathrm{G}$ at a certain growth rate. The maximum $\mathrm{G}$ value starts from $2.5 \mathrm{G}$, increasing at the rate of $0.5 \mathrm{G} / \mathrm{r}$, until the subject gets to the endurance end. The doctor real-time monitors ear pulse, ECG and other physiological signals, and comprehensively judges if the subject gets to the endurance end, according to the level state of ear pulse, combining with the subjective narration of peripheral lamp disappeared or central lamp indistinct and the expression of the subject. EEG data when running, and before and after running is recorded by using a portable EEG recorder. Electrode signal of 16 leads is recorded in accordance with the electrodes positions of 10-20 system of international EEG standard electrodes installation method. All electrodes take the electrode at ipsilateral earlobe (A1 or A2) as the reference electrode [23]. Subject is worn of different types of fasten mesh cap, and each electrode is affixed special medical tape to prevent becoming loose in running. In experiment the subject is required to try to keep the natural state. Measuring method of single electrode lead is selected. The sampling rate is $128 \mathrm{~Hz}$.

\section{B. Experiment results and analysis}

For EEG data of each lead of 16 leads, the EEG data of $10 \mathrm{~s}$ at quiet state that is when $1 \mathrm{G}$ and under $+\mathrm{Gz}$ acceleration are selected separately and is analyzed by using Hilbert-Huang transformation. FFT spectrum graph, margin spectrum, EMD decomposition graph and HHT spectrum graph of Fp1 lead at quiet state that is when $1 \mathrm{G}$ and when $3 \mathrm{G}$ are made, and are analyzed combining with FFT amplitude frequency graph. The following Figure1 is FFT amplitude frequency diagram of EEG Fp1 lead at quiet state that is when 1G.Figure2 Figure4 are marginal spectrum diagram, EMD decomposition diagram and HHT spectrum diagram of Fp1 lead when 3G by HHT analysis. Figure5 is FFT amplitude frequency diagram of Fp1 lead when 3G. Figure6 Figure8 are marginal spectrum diagram, EMD decomposition diagram and HHT spectrum diagram of Fp1 lead when $3 \mathrm{G}$ by HHT analysis.

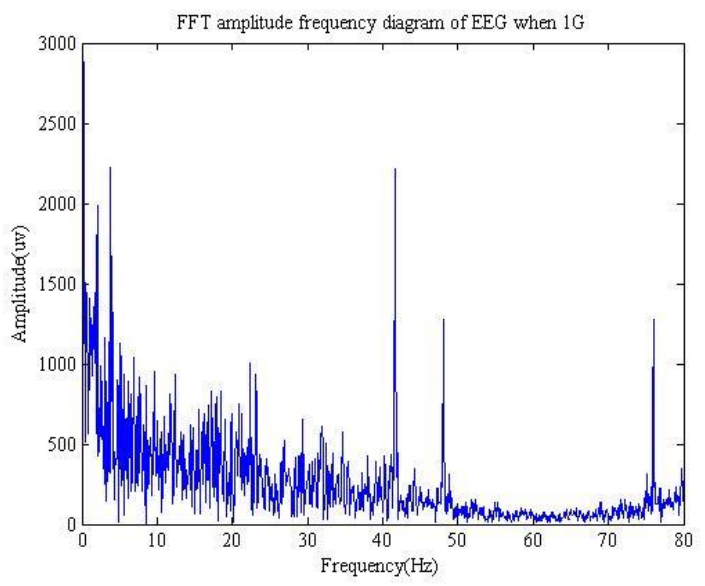

FIGURE I. FFT AMPLITUDE FREQUENCY DIAGRAM OF EEG FP1 LEAD WHEN $1 \mathrm{G}$

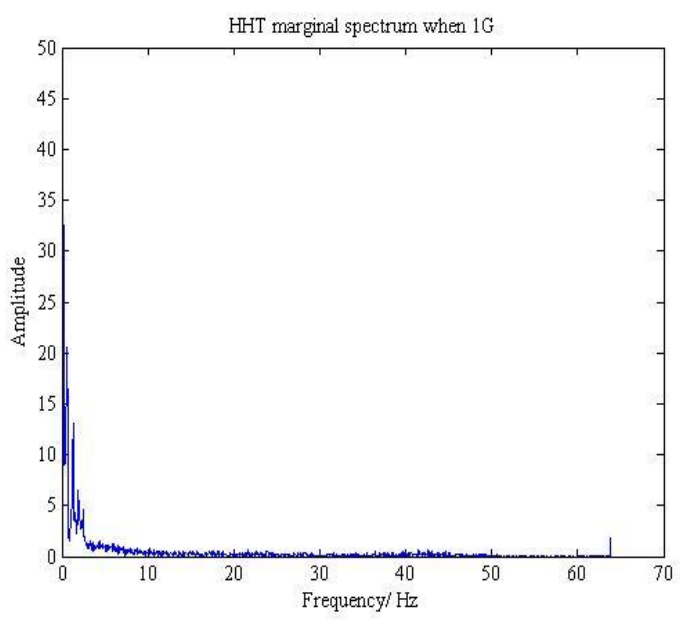

FIGURE II. HHT MARGINAL SPECTRUM DIAGRAM OF EEG FP1 LEAD WHEN $1 \mathrm{G}$

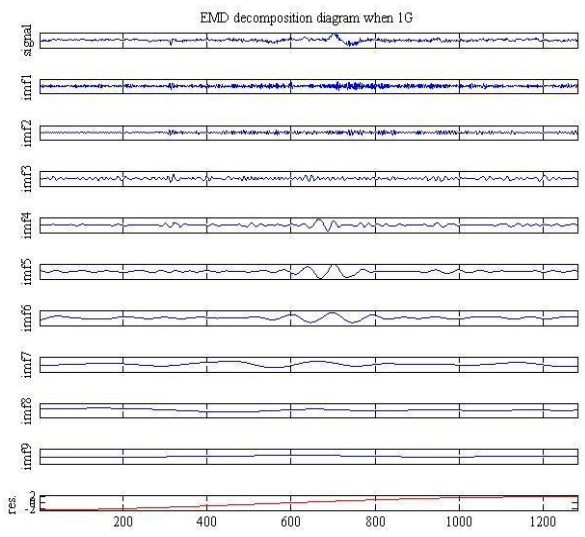

FIGURE.III. EMD DECOMPOSITION DIAGRAM OF EEG FP1 LEAD WHEN $1 \mathrm{G}$ 


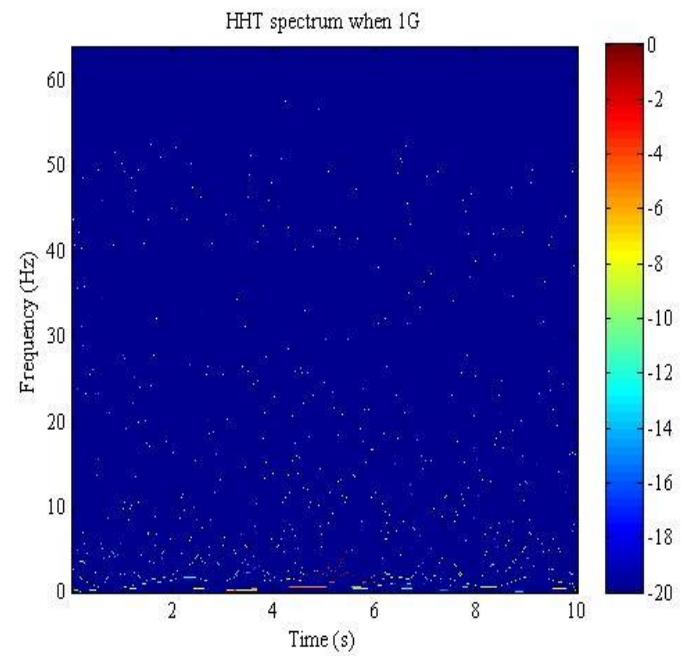

FIGURE.IV. HHT SPECTRUM DIAGRAM OF EEG FP1 LEAD WHEN 1G

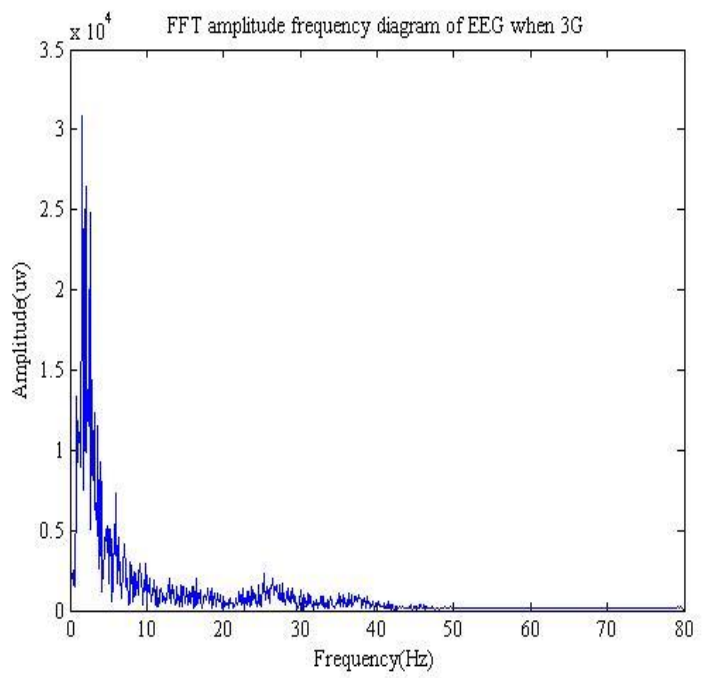

FIGURE.V FFT AMPLITUDE FREQUENCY DIAGRAM OF EEG FP1 LEAD WHEN $3 \mathrm{G}$

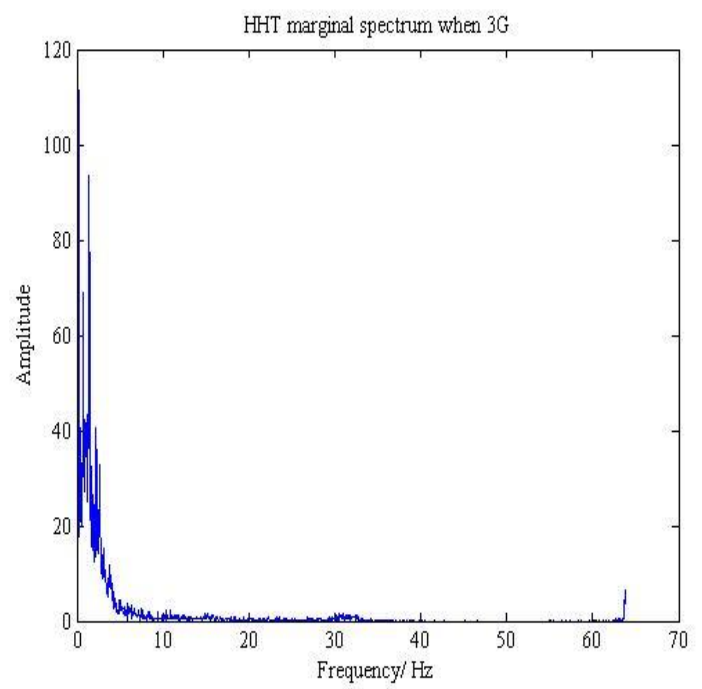

FIGURE.VI. HHT MARGINAL SPECTRUM DIAGRAM OF EEG FP1 LEAD WHEN 3G

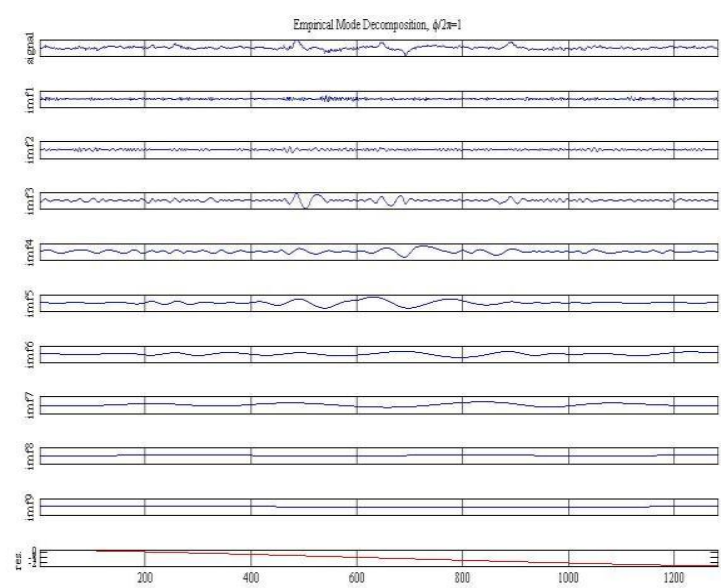

FIGURE VII EMD DECOMPOSITION DIAGRAM OF EEG FP1 LEAD WHEN 3G

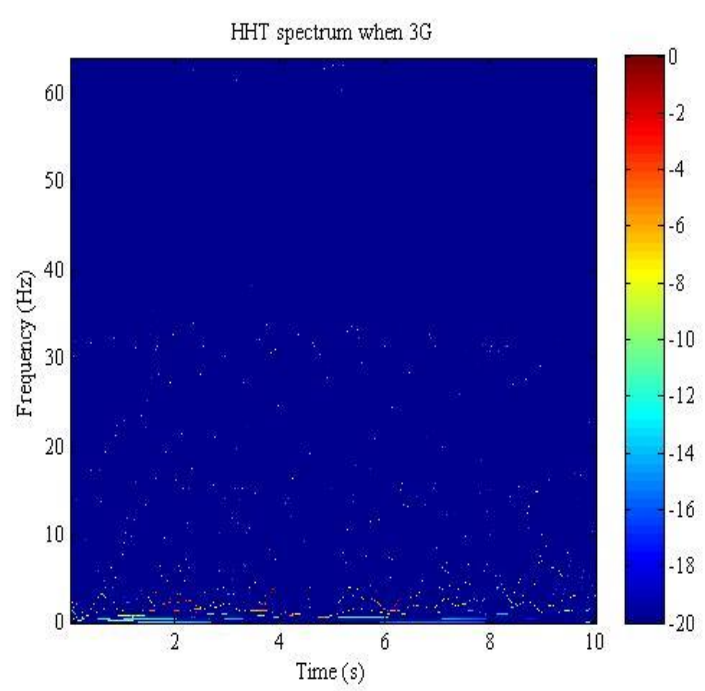

FIGURE VIII $\backslash$. HHT SPECTRUM DIAGRAM OF EEG FP1 LEAD WHEN $3 \mathrm{G}$

From the graphs, it can be seen that EEG both has obvious change and signal energy of all frequency bands has increasing of different degrees under $+\mathrm{Gz}$ acceleration no matter for FFT analysis or for HHT analysis. The energy and amplitude of low frequency components in EEG signal has significant increasing under $+\mathrm{Gz}$ acceleration relatively to other wave bands, especially the low frequency component below $10 \mathrm{~Hz}$ increases significantly and the amplitude is relatively larger, mainly concentrated in delta and theta frequency band, while high frequency component more than $50 \mathrm{~Hz}$ is rare and the amplitude is relatively close to 0 . Almost all sampling points appear a small peak at $65 \mathrm{~Hz}$, and more closer to the occipital, more great the strength of peak is. Analyzing reason, the frequency of power interference wave is around $50 \mathrm{~Hz}$, and the frequency of instruments and other electromagnetic interference waves generally is less fixed, explaining that under $\mathrm{G}$ load, the high frequency components of EEG reduced significantly, while low frequency components increase, especially the appeared peak at $65 \mathrm{~Hz}$ 
explains that human body can produce a characteristic wave which frequency is about $65 \mathrm{~Hz}$, and this characteristic wave reflects more obviously in the marginal spectrum graph of HHT. From the graph, it can be learned that Fourier transformation can not reflect the time characteristic of signal, so it is limited using it to analyze the non-stationary signal which frequency is transient with time. The frequency in FFT spectrum diagram represents the exited frequency of signal in the whole time process, the peak value represents the peak value of the frequency which is global existence in signal, and the frequency of marginal spectrum is instantaneous frequency, the peak value which corresponds to represents the energy sum the instantaneous frequency appeared in this signal corresponds to. Some scholars have pointed out that when stability degree is zero, Fourier spectrum and marginal spectrum of signal have the same concept.

It can be seen from Figure 3 and Figure 7, the first 6 order IMF components have more obvious fluctuation phenomena, the behind a few order IMF components tend to be gentle, which means that the former several order IMF components namely the middle and high frequency components in EEG account for the main component, so first several order IMF components reflect the characteristic of middle and high frequency parts of EEG transient response, while the behind several order IMF components reflect the characteristic of low frequency part. It can be seen from graphs, EMD can more accurately decompose complex signal into the intrinsic mode function from high frequency to low frequency, so for the actually existed various kinds of non-stationary signals, such as EEG signal, they can be analyzed by using this advantage of EMD, and signal is decomposed into the a superposition of several stable IMFs from high frequency to low frequency and residual signals, then the decomposed out IMF is made spectrum graph analysis by using Hilbert transformation. The responsive Hilbert spectrum is got by making Hilbert transformation for the original signal. When subjected to outside pulse action, the shown frequency is more abundant, the energy of all frequency bands has increasing of different degrees, especially the energy of delta and theta frequency band increases obviously. Compared to the static state, points distribution in HHT spectrum figures under the action of $G$ load also changes from large area scattering to being intensive at the low-frequency part, especially points concentrated in delta and theta frequency bands are more, indicating that energy transfers from high frequency to low frequency, which is basically consistent with information of energy variation shown out by FFT. But the frequency resolution ratio of FFT is lower, and the change of density degree of high and low frequency appeared in Hilbert spectrum can not be displayed by Fourier spectrum. In empirical mode decomposition (EMD), a few obvious fluctuation phenomena can also be seen, and in Hilbert spectrum it is shown at several places where high frequency more than $30 \mathrm{~Hz}$ appears, but likewise, the proportion of high frequency accounts for is not big, mainly dominated by the middle and low frequency, reflecting the characteristic of EEG itself. The processing effect of Fast Furious transformation for non stationary, nonlinear signal is not satisfactory. Furthermore, according to Heisenberg uncertainty principle, the product of time width and band width of signal should be a constant relevant to sampling rate, so if time accuracy doubles, frequency resolution ratio will become 2 times of the original, not achieving the original precision. For EEG signal, resolution ratio of frequency can not be too low, at least accurately at $0.5 \mathrm{~Hz}$, so when resolution ratio of frequency is required, the time window length is very likely to appear too large, at this time FFT Fourier transformation may be difficult to meet the requirement. While Hilbert Huang transformation is calculation method of instantaneous frequency energy, well giving consideration to the requirement of time resolution ratio and frequency resolution ratio, and the basis function of this method has characteristic of self - adaptive, more suitable for the analysis and processing of non stationary signal. It can help to more clearly understand the distribution of low and high frequency part in signal, more deeply to understanding the nature of signal, and identify the characteristic of signal. Of course, this method also needs to be studied and perfected continuously in practical application.

\section{CONCLUSIONS}

This study introduces the basic principle and method of HHT, and induces HHT into the application and study into the dynamic EEG data under $+\mathrm{Gz}$ acceleration. For EEG data at static state that is when $1 \mathrm{G}$ and dynamic EEG data under $+\mathrm{Gz}$ acceleration, spectrum figures are made out by using HHT method and are made time frequency analysis, and the basic change of different frequency bands in EEG is found, that is, signal energy of each frequency band has increasing of different degrees, and the energy and the amplitude of low frequency component all has significant increasing relatively to other wave bands, especially the low frequency component below $10 \mathrm{~Hz}$ increases significantly and the amplitude is relatively more large, and each point appears a small peak at $65 \mathrm{~Hz}$, more closer to the occipital position, more greater the strength of peak is, indicating that under the action of high $G$ load human will produce a characteristic wave which frequency is about $65 \mathrm{~Hz}$. Some analysis results of HHT are basically consistent with the analysis results of FFT, proving the feasibility of this method in analysis of dynamic EEG data. At the same time it is explained that, the basis function of HHT method has self-adaptive characteristic, and is more suitable for analysis and processing of non stationary signal. HHT method can not only separate the low frequency and high frequency part in transient signal by EMD decomposition, but also the marginal spectrum and time-frequency spectrum got by Hilbert transformation can better display the distribution of signal energy in time frequency domain, can more clearly understand the distribution of low and high frequency part in signal, more clearly reflect frequency spectrum change characteristic of dynamic EEG, and help to better identify characteristic of signal. Of course, the development and improvement of any kind of signal requires a process, so HHT method still needs to be further studied and improved in practical application.

\section{REFERENCES}

[1] Koji Morikawa, Akinori Matsumoto, Shrishail Patki, Bernard Grundlehner,Auryn Verwegen, Jiawei Xu, Srinjoy Mitra and Julien Penders. Compact Wireless EEG Sys tem with Active Electrodes for 
Daily Healthcare Monitoring.IEEE International Conference on Consumer Electronics (ICCE).2013:204-205.

[2] Jiawei Xu, Refet Firat Yazicioglu,Bernard Grundlehner, Pieter Harpe, Kofi A. A. Makinwa, and Chris Van Hoof. A $160 \mu$ W 8-Channel Active Electrode System for EEG Monitoring. IEEE TRANSACTIONS ON BIOMEDICAL CIRCUITS AND YSTEMS.2011;5(6):555-567.

[3] SN. Tang, FC. Jan, HW. Cheng, CK. Lin, G Z. Wu. Multimode Memory-Based FFT Processor for Wireless Display FD-OCT Medical Systems. IEEE Transactions on Circuits and Systems I: Regular Papers.2014; 61 ( 12): $3394-3406$.

[4] Jing Han, Lingling Zhang, G. Leus. Partial FFT Demodulation for MIMO-OFDM Over Time-Varying Underwater Acoustic Channels. IEEE Signal Processing Letters.2016: 23 (2): 282 - 286.

[5] T. Yang,H. Pen, Z. Wang, C. S. Chang. Feature Knowledge Based Fault Detection of Induction Motors Through the Analysis of Stator Current Data. IEEE Transactions on Instrumentation and Measurement.2016 ;65 ( 3 ): 549 - 558.

[6] C. Thirumoorthi, T. Karthikeyan. Easy optimization of image transformation using sFFT algorithm with HALIDE language. 2014 International Conference on Contemporary Computing and Informatics (IC3I), Mysore, 2014: 1188 - 1190.

[7] K. Kumar, N. Mustafa, J.-P. Li, R.-A. Shaikh, S.-A. Khan, A.Khan, Image edge detection scheme using wavelet transform, 2014 11th International Computer Conference on Wavelet Active Media Technology and Information Processing (ICCWAMTIP), Chengdu, 2014:261 - 265

[8] R. Mothi, M. Karthikeyan, A wavelet packet and fuzzy based digital image watermarking, 2013 IEEE International Conference on Computational Intelligence and Computing Research (ICCIC), Enathi, 2013:1 - 5.

[9] J.S Pan, and L. Yue, "A ridge extraction algorithm based on partial differential equations of the wavelet transform, "2014 IEEE Symposium on Computational Intelligence for Multimedia, Signal and Vision Processing (CIMSIVP), Orlando, FL, pp.1 - 5, 2014.

[10] G. Quellec, M. Lamard, G. Cazuguel, B. Cochener, and C. Roux, "Fast Wavelet-Based Image Characterization for Highly Adaptive Image Retrieval, "IEEE Transactions on Image Processing, vol.21(4), 2012,pp.1613 - 1623 .

[11] S.A.R. Naqvi, I. Touqir, and S.A. Raza, "Adaptive geometric wavelet transform based two dimensional data compression, "2013 7th IEEE GCC Conference and Exhibition (GCC), Doha , pp.583 - 588, 2013.

[12] N. Fuad, W. Mansor, and K.Y. Lee, "Wavelet packet analysis of EEG signals from dyslexic children with writing disability," in: 35th Annual International Conference of the IEEE, Engineering in Medicine and Biology Society (EMBC), Osaka, pp.2890-2893, 2013.

[13] K. Thirumala, A.C. Umarikar, and T. Jain, "Estimation of Single-Phase and Three-Phase Power-Quality Indices Using Empirical Wavelet Transform," IEEE Transactions on Power Delivery, vol.30(1),2014, pp.445-454.

[14] G. Serbes, N. Aydin, and H.O. Gulcur, "Directional dual-tree complex wavelet packet transform, "2013 35th Annual International Conference of the IEEE, Engineering in Medicine and Biology Society (EMBC), Osaka, pp.3046-3049, 2013.

[15] S. Bharkad, and M. Kokare, "Fingerprint matching using discreet wavelet packet transform, "2013 IEEE 3rd International Advance Computing Conference (IACC), Ghaziabad, pp.1183-1188, 2013.

[16] K. Rai, V. Bajaj, A. Kumar. Hilbert-Huang transform based classification of sleep and wake EEG signals using fuzzy c-means algorithm. 2015 International Conference on Communications and Signal Processing (ICCSP), IEEE, Melmaruvathur, 2015: 0460 - 0464.

[17] Y. Wang, C. Chen, H. He. Large Eddy Simulation and Hilbert Huang Transform for fluctuation pressure of high speed train. 2015 IEEE International Instrumentation and Measurement Technology Conference (I2MTC), Pisa, 2015: $284-288$

[18] Rahul Kumar Chaurasiya,K. Jain, S. Goutam,Manisha. Epileptic seizure detection using HHT and SVM. 2015 International Conference on Electrical, Electronics, Signals, Communication and Optimization (EESCO), Visakhapatnam, 2015: 1-6.
[19] K. Y. Lin, D. Y. Chen, W. J. Tsai. Face-Based Heart Rate Signal Decomposition and Evaluation Using Multiple Linear Regression. IEEE Sensors Journal .2016; 16 ( 5 ): 1351 - 1360.

[20] M. MoeinDarbari , H. Pourreza , M. Monfared. Design of an automated system for detection and classification of power quality disturbances. 2015 5th International Conference on Computer and Knowledge Engineering (ICCKE), Mashhad, 2015: 181 - 186.

[21] B. Lenka . Time-frequency analysis of non-stationary electrocardiogram signals using Hilbert-Huang Transform. 2015 International Conference on Communications and Signal Processing (ICCSP), Melmaruvathur, 2015: 1156 - 1159 .

[22] H.N.Lian,L.L.Xie. Application of three transformations in the analysis of in strong earthquake records. NORTH CHINA EARTHQUAKE SCIENCES, 2007,25(3):28-33.

[23] M. Nakamura, Q. Chen, T. Sugi, A .Ikeda, H. Shibasaki, Technical quality evaluation of EEG recording based on electroencephalographers' knowledge, Medical Engineering \& Physics, 2005, 27:93-100. 